\title{
Perinatal Mortality in Term and Preterm Twin and Singleton Births
}

\author{
Jennifer C. Payne', M. Karen Campbell ${ }^{2,3,4,5}$, Orlando DaSilva ${ }^{3,2}$, and John Koval ${ }^{2}$ \\ I Surveillance and Risk Assessment Division, Centre for Chronic Disease Prevention and Control, Health Canada, Ottawa, Ontario, Canada \\ ${ }^{2}$ Department of Epidemiology and Biostatistics, The University of Western Ontario, London, Ontario, Canada. \\ ${ }^{3}$ Department of Paediatrics, The University of Western Ontario, London, Ontario, Canada. \\ ${ }^{4}$ Department of Obstetrics and Gynaecology, The University of Western Ontario, London, Ontario, Canada. \\ ${ }^{5}$ Lawson Health Research Institute, London, Ontario, Canada
}

\begin{abstract}
Ithough, in general, twins have higher perinatal mortality Arates than singletons, preterm twins have lower perinatal mortality rates than singletons of the same birth weight or gestational age. This study investigated the hypotheses that this paradoxical twin advantage: 1) is due to gestational age distribution differences between the singleton and twin populations, and 2) is due to increased likelihood of birth having occurred in a tertiary perinatal center. A pre-existing, time-limited data set of all births in the province of Ontario in odd years between 1979 and 1985 was chosen for this study because of the large sample size ( $n=618,579)$. Multivariable logistic regression of the relationship between perinatal mortality and twin status was controlled for mother's age, hospital level and gestational age. Findings confirm the lower mortality of preterm twins. After controlling for level of hospital of birth this difference remained, suggesting that level of hospital of birth was not a major factor responsible for the twin advantage. Analyses in which gestational age was standardized indicate that, for those whose gestational age was less than $2 S D$ below the mean for their particular group (twin or singleton), twins were actually at higher risk than singletons. These results support hypothesis 1 and do not strongly support hypothesis 2 . The results also support earlier authors' suggestions that the definition of term birth should be different for twins and singletons
\end{abstract}

Twins have a perinatal mortality rate four to 10 times higher than singletons (Campbell, 1993; Kiely, 1990; Marvate \& Norman, 1982;) and are more likely to experience adverse long-term outcomes such as cerebral palsy (Williams et al., 1996). The higher perinatal mortality and morbidity in twins can largely be explained by their higher incidence of preterm birth and low birth weight. As well, some twins have additional risk arising from situations unique to multiple birth such as whether the twins are monoamniotic, monochorionic, discordant and whether twin-to-twin transfusion syndrome has occurred (D'Alton \& Simpson, 1995). Paradoxically, although singletons have a lower perinatal mortality rate overall, preterm twins ( $<37$ weeks gestational age) actually have lower gestational age-specific mortality rates compared to singletons (Buekens \& Wilcox, 1993; Kiely, 1998; Kilpatrick et al., 1996; Minakami \& Sato, 1996; Payne, 1997).

The objective of the study was to investigate two hypotheses which could explain the apparent twin advantage at low gestational ages. Two specific hypotheses were examined: 1) that the apparent twin advantage is partially a result of gestational age distribution differences between the singleton and twin populations; and 2) that this advantage is partially conferred by increased likelihood of birth in a tertiary care hospital for twins as compared to singletons.

\section{Materials and Methods}

The data for this study were obtained from the Perinatal Information System at the Ontario Ministry of Health. The data set was constructed for a specific purpose unrelated to this study and was limited to odd years between 1979 and 1987. For those years, data on all reported births in the province of Ontario were included. Anonymous singleton and twin birth certificate records of the Registrar General were linked to death certificate records (restricted to death at age $<28$ days) and Hospital Medical Records Institute (HMRI) hospital discharge data (Campbell, 1993). Although a decade old, this database was chosen because it provided a reasonably large number of twin cases on which to base parameter estimates. However, since it contained few variables, some covariates of interest were not available for study. Further details may be found in Payne (1997).

Data were managed and analyzed using the computer programs SAS and BMDP. One twin from each pair was chosen at random (using SAS PROC RANUNI) for the sample. This avoided correlation between subjects and the resulting loss of control over $p$-values which would occur if both members of the twin pairs were in the sample. The two members of each twin pair were identified based on common values for the shared birth variables of twin status, birthplace, mother's age and marital status, location of mother's residence (municipality, census division and region) and gestational age.

The outcome of interest was death during either the perinatal or late neonatal period. The reason for including late neonatal deaths was as follows. There was a disproportionate reduction in early neonatal deaths, and a shift of some of these deaths to the late neonatal period ( $\geq 7$ days, < 28 days), for term births in Ontario between 1979 and 1987 (Campbell \& Webster, 1993). Since the timing of

Address for correspondence: Dr M. Karen Campbell, Departments of Epidemiology and Biostatistics, Obstetrics and Gynaecology and Paediatrics, Kresge Building, University of Western Ontario, London Ontario, Canada. N6A 5C1. Email: campbell@biostats.uwo.ca 
neonatal death (early versus late) was gestational age related, basing the comparison on a mortality rate that included the late neonatal period reduced the possibility of bias. All covariates were categorical. Mother's age was collected as a continuous variable but, because of computational difficulties with convergence in the computer program, age was categorized. The number of twins was insufficient to permit three strata and so the variable was dichotomized ( $<30$ years, $\geq 30$ years).

Contingency tables and chi-square analysis were used to study the association between twin versus singleton status and mortality within gestational age strata. Multivariable comparison of factors affecting singleton and twin perinatal mortality, employed logistic regression using SAS PROC LOGISTIC and BMDPLR.

In order to address the first hypothesis, gestational age distributions were examined using two different stratifications: i) stratification based on absolute gestational age (the traditional "high risk" definition for gestational age is $<37$ weeks); ii) stratification based on standardized gestational age using the method of Rooth (1980) where standardization was done within each group separately (singleton or twin) to correct for distributional differences (the "high risk" category are those less than $-2 S D$ ). Comparison of these two sets of results would provide support for or against the first hypothesis. Twin versus singleton status was the primary risk factor of interest, and the possible confounders were mother's age and gestational age. The second hypothesis was explored through examination of the influence of hospital level (community or district hospital vs. tertiary hospital) in the logistic model.

Each logistic regression model was fitted in two stages (Kleinbaum, 1988). At each, backward elimination from the full model was used to determine which variables could be removed from the model. In stage one, the full model included first-order interaction terms of "hospital level", mothers age and gestational age with twin versus singleton status. All main effects were forced in. A significance level of 0.05 was used to determine any significant interactions. If an interaction remained in the model after stage one, then the data was split over levels of the covariate that interacted with twin status. At the start of stage two, no interactions remained in the model; the full model consisted of all remaining main effects. All main effects were sequentially removed from the model if their $p$-value remained above 0.15 . The elimination criterion was set at 0.15 because this has been shown to be an appropriate sig- nificance level for forward regression (Lee \& Koval, 1997); no similar assessment has been done for backward elimination. The need to set the criterion for variable selection at a level above 0.05 was established by Mickey and Greenland (Mickey \& Greenland, 1989). Although the variable selection criterion was 0.15 , statistical significance was set at 0.05 .

\section{Results}

The study sample included 618,579 births - 612,786 singletons and 5793 twin pairs. A further 594 twin pairs were excluded because they could not be matched with their sibling with certainty. The mean gestational ages were 39.4 and 36.6 weeks for singletons and twins, respectively, with corresponding inter-quartile ranges (25th to 75 th percentile) of 39 to 40 and 35 to 39 weeks.

Table 1 presents perinatal mortality rates stratified by gestational age ( $<37$ weeks and $\geq 37$ weeks). The proportion of births which occur preterm ( $<37$ weeks) was almost eight times as great for twins as compared to singletons and the preterm twins had a lower mortality rate. This is reversed in the term ( $\geq 37$ weeks) category where twins were under-represented in total births and mortality was higher in twins than in singletons.

Table 2 presents the results of the standardized approach to gestational age, dichotomizing on statistical grounds at $-2 S D$. The distributions of total births for twins and singletons were more similar; however twins $<-2$ $S D$ are still disproportionately represented. This suggests that the twin gestational age distribution is shifted to the left of the singleton distribution and also has a larger left tail. Thus, even after standardizing for distribution, twins are still over-represented in the highest risk category $(<-2$ $S D)$. Further, the twin mortality rate is higher than singletons in both the high risk $(<-2 S D)$ and the low risk categories.

Tables 3 and 4 present multivariable analyses using the two approaches to gestational age stratification. Due to a significant statistical interaction between twin status and gestational age, different models were fitted in each stratum which resulted in different covariates selected by the model for each stratum. The results in Table 3 show that preterm twins still have lower perinatal mortality after control for level of birth hospital. In Table 4, after controlling for significant covariates, twin mortality was greater than singleton mortality both for those whose gestational age was $<-2 S D$ as well as for those $\geq-2 S D$.

\section{Table 1}

Distribution and Mortality Rates of Preterm and Term Singleton and Twin Births

\begin{tabular}{lcccc}
\hline \multirow{2}{*}{ Gestational Age } & \multicolumn{2}{c}{ Singletons } & \multicolumn{2}{c}{ Twins } \\
& Number of Births (\%) & Deaths* number (rate) & Number of Births (\%) & Deaths*number (rate) \\
\hline Preterm (<37 weeks) & 32,325 & 3,195 & 2,207 & 140 \\
& $(5.3 \%)$ & $(98.8 / 1000)$ & $(38.1 \%)$ & $(63.4 / 1000)$ \\
Term ( $\geq 37$ weeks) & 580,461 & 2,050 & 3,586 & 32 \\
& $(94.7 \%)$ & $(3.5 / 1000)$ & $(61.9 \%)$ & $(8.9 / 1000)$ \\
\hline
\end{tabular}

Note: * Deaths include fetal deaths which occurred after 20 weeks gestation and infant deaths $<28$ days after livebirth. 
Table 2

Distribution and Mortality Rate of Singleton and Twin Births Which Are $<-2 S D$ and $\geq 2 S D$ of Gestational Ages Observed

\begin{tabular}{lcccc}
\hline \multirow{2}{*}{$\begin{array}{c}\text { Standardized Gestational Age } \\
\text { Number of Births (\%) }\end{array}$} & Deaths*number (rate) & Number of Births (\%) & Deaths*number (rate) \\
\hline$<-2 S D$ & 18,284 & 2,996 & 352 & 102 \\
& $(3.0 \%)$ & $(163.9 / 1000)$ & $(6.1 \%)$ & $(289.8 / 1000)$ \\
& $5-2 S D$ & 2,249 & 5441 & 70 \\
& $(99,502$ & $(3.8 / 1000)$ & $(93.9 \%)$ & $(12.9 / 1000)$ \\
\hline
\end{tabular}

Note: Singleton GA standardized against singletons and twin GA standardized against twins; - $2 S D$ corresponds to 35.5 weeks gestational age for singletons and 30.1 weeks gestational age for twins.

${ }^{*}$ Deaths include fetal deaths which occurred after 20 weeks gestation and infant deaths $<28$ days after livebirth.

\section{Table 3}

Multivariable Logistic Regression of Mortality in the Perinatal or Late Neonatal Period for Births Which Are Preterm and Term

\begin{tabular}{llc}
\hline & FACTOR & $\begin{array}{c}\text { OdDS RATIO } \\
\text { (95\% CONFIDENCE INTERVAL) }\end{array}$ \\
\hline Preterm $(<37$ wks) & Twin & $0.61(0.51,0.73)$ \\
& Tertiary Hospital & $1.11(1.02,1.21)$ \\
Term $(\geq 37$ wks $)$ & Twin & $2.52(1.77,3.57)$ \\
& Maternal Age & $1.13(1.03,1.24)$ \\
\hline
\end{tabular}

Note: Covariates were included only if statistically significant.

Analyses were stratified by gestational age due to a statistically significant interaction between gestational age and twin status.

The sample included all reported twin and singleton births in Ontario in odd numbered years between 1979 and 1987.

\section{Table 4}

Multivariable Logistic Regression of Mortality in the Perinatal or Late Neonatal Period for Births Which Are $<-2 S D$ or $\geq 2 S D$ of Gestational Ages Observed

\begin{tabular}{llc}
\hline Stratum & Factor & $\begin{array}{c}\text { Odds Ratio } \\
\text { (95\% CONFIDENCE INTERVAL) }\end{array}$ \\
\hline Gestational age & Twin & $2.25(1.77,2.84)$ \\
$<-2 S D$ & Tertiary Hospital & $0.74(0.68,0.81)$ \\
Gestational age & Twin & $3.41(2.68,4.33)$ \\
$\geq-2 S D$ & Maternal Age & $1.10(1.01,1.20)$
\end{tabular}

Note: Covariates were included only if statistically significant.

Gestational age was standardized relative to the distribution of gestational ages separately for twins and singletons.

Analyses were stratified due to a statistically significant interaction between standardized gestational age and twin status.

The sample included all reported twin and singleton births in Ontario in odd numbered years between 1979 and 1987.

\section{$\overline{\text { Discussion }}$}

This study confirmed that, at younger gestational ages, twins have a lower mortality rate than singletons. Support for hypothesis 1, that the twin advantage at lower gestational ages is a product of distributional differences, was shown in this study. Our results, and those from other studies of perinatal mortality (Buekens \& Wilcox, 1993; Joseph et al., 2001; Kiely, 1998; Kilpatrick et al., 1996; Minakami \& Sato, 1996; Payne, 1997), suggest that twins are further along in their gestational course than singletons of the same gestational age. The twin advantage disappears at gestations near term, and twins near term are at a disadvantage relative to singletons. When analyses were repeated using standardized gestational age distributions, the lower gestational age stratum showed a better outcome for singletons. This was caused, at least in part, by the fact that the twin infants in each stratum are younger than their singleton counterparts. Hertz-Picciotto and Din-Dzietham (1998), in their study comparing African-American and European-American infants in North Carolina, emphasized the advantage of using percentile-transformed gestational age when comparing risks in unlike populations. Our study suggests that percentile transformation may be an approach to assessing mortality risk for twins.

Since distributional differences explain some of the advantage twins have at lower gestational ages, it is tempting to speculate that twins "mature" faster than singletons. It has been suggested that lung maturation occurs earlier in twins but reaches a plateau later in gestation (Dhall \& Majumdar, 1986; Leveno et al., 1984). Similarly, it has been shown that brain maturation, as measured by brainstem evoked auditory responses, is accelerated by as much as 3 to 4 weeks in small for gestational age newborns including those from multiple pregnancies (Amiel-Tison \& Pettigrew, 1991). As well, Blickstein (2001a,b) has reported evidence that fetal growth in twins ceases beyond 38 weeks gestation and that the risk of cerebral palsy in twins decreases with increasing gestational age until 37-38 weeks and then increases again. Blickstein has drawn evidence from a variety of measures of maturity to suggest that term for twins may in fact be 37-38 weeks. The possible mechanism for earlier maturity in twins may be the effects of glucocorticoids, other steroid hormones and catecholamines which have been shown to be elevated in pregnancies with placental dysfunction (Amiel-Tison \& Pettigrew, 1991). This may provide a possible explanation for the lower gestational age specific perinatal mortality in twins relative to singletons at 31-36 weeks gestation but the higher twin mortality at term. However, more data on morbidity for twins $\leq 36$ weeks gestation is needed. Even in terms of mortality, the twin advantage disappears below 28 weeks gestation (Donovan et al., 1998).

Our analysis included births in the province of Ontario in odd years from 1979 to 1987 . Recent analyses of Canadian statistics from 1994-1996 relative to 1985-1987 suggest that gestational ages for twin births have decreased 
due to an increase in twin births at 34-36 weeks gestation (Joseph et al., 2001) presumably due to increased induction at those gestational ages to avoid the previously documented increase in stillbirths among twins > 38 weeks gestation. This pattern of increased birth at 34-36 weeks gestational age coincides with a trend of decrease in twin perinatal mortality risk relative to singletons at those gestational ages. Presumably our analyses, pre-dating this trend, more closely reflect the natural timing of spontaneous twin birth and provide a basis for testing the hypothesis that distributional differences are important in explaining the twin advantage. The more recent data reported by Joseph et al. illustrate the improved outcomes which can be seen with increased induction just prior to term.

These analyses focused on gestational age and not birthweight. It is known that birthweight is also an important determinant of perinatal mortality that is highly correlated with gestational age. It is unlikely that birthweight differences would explain the paradoxically better twin outcomes at lower gestations since twins would be, on average, lighter than singletons of the same gestational ages thus presumably being of higher risk for that reason. A separate analysis of birthweight has been done (Payne, 1997) and, as with gestational age, it is clear that there is a distributional difference between twins and singletons with lighter twins having better mortality outcomes than sameweight singletons.

The findings of our study did not strongly support our second hypothesis that the twin advantage was partially the result of increased likelihood of birth in a tertiary center. Specifically, Table 3 showed that, after controlling for hospital level and before controlling for distributional differences, low gestational age twins still have a lower perinatal mortality rate than singletons regardless of level of hospital of birth; the magnitude of the advantage was not influenced by place of birth.

Place of birth was shown to have an independent effect on the mortality of preterm twins and singletons. For those $<37$ weeks gestational age, those born in a community or district hospital had the lowest mortality and birth in a tertiary center was associated with higher perinatal mortality. This is easily explained by referral patterns. Even among those $<37$ weeks gestation, there would be a difference in the gestational age distributions with the youngest and those at greatest risk were the most likely to have been transferred during labor to the tertiary hospital (Campbell et al., 1991). The pattern was quite different for those in the $<-2 S D$ groups that consisted of the very youngest (Table 4) twins and singletons. For these highest risk infants, being born in a tertiary hospital was associated with a lower mortality rate. Thus, although our data show that place of birth is not likely a major explanation of the twin advantage at lower gestational ages, our data also support earlier work illustrating that the highest risk births have better outcomes when birth occurs at a tertiary center.

While it is clear that standardization eliminated the paradoxically better outcomes among twin births, thus strongly supporting hypothesis 1 , the etiology of the effect is not clear. It is recommended that further studies be conducted which include variables necessary to better understand the etiology of this effect. These variables would include twin zygosity, chorionicity and maternal risk factors that have been associated with perinatal outcome such as smoking, diabetes, hypertension, urinary tract infection within 2 weeks of delivery and history of preterm delivery, low birth-weight or neonatal death. A future study would ideally also use a database that would provide a larger twin sample. This would result in more precise parameter estimates. Further, it would provide an opportunity to stratify maternal age into additional categories which would permit investigation of the effect of maternal age (both the effect of "younger" and "older" mothers) on the associations identified in this study.

In the meantime, we conclude that gestational age distributional differences largely account for the paradoxical twin advantage observed at lower birth weights and younger gestational ages. Other authors have recommended serious reconsideration of the definition of "term" in twins based on their analyses of perinatal mortality in the US (Kiley, 1998) and in Japan (Minakami \& Sato, 1996) as well as analyses of fetal growth and infant morbidity (Blickstein, 2001a,b). Our analyses of Ontario perinatal mortality data support this recommendation.

\section{References}

Amiel-Tison, C., \& Pettigrew, A. G. (1991). Adaptive changes in the developing brain during intrauterine stress. Brain Development, 13, 67-76.

Blickstein, I. (2001a). Controversial issues in the management of multiple pregnancies. Twin Research, 4, 165-167.

Blickstein, I. (2001b). When and how to deliver twins. The second world congress on controversies in obstetrics, gynecology and infertility (pp. 93-98). Italy: Monduzzi Editore.

Buekens, P. \& Wilcox, A. (1993). Why do small twins have a lower mortality rate than small singletons? American Journal of Obstetrics and Gynecology, 168, 937-941.

Campbell, M. K. (1993). Perinatal mortality in Ontario in 1987: Time trends, determinants and implications. Ontario Ministry of Health Report (catalog \# 2225218). Toronto: Queen's Park Printer for Ontario.

Campbell, M. K., Chance, G. W., Natale, R., Dodman, N., Halinda, E., \& Turner, L. (1991). Is perinatal care in Southwest Ontario regionalized? Canadian Medical Association Journal, 144, 305-312.

Campbell, M. K., \& Webster, K. M. (1993). Age at neonatal death in Ontario, 1979-1987: Implications for the interpretation of mortality markers. Paediatric and Perinatal Epidemiology, 7, 426-433.

Donovan, E. F., Ehrenkranz, R. A., Shankaran, S., Stevenson, D. K., Wright, L. L., Younes, N., et. al (1998). Outcomes of very low birth weight twins cared for in the National Institute of Child Health and Human Development Neonatal Research Network's intensive care units. American Journal of Obstetrics and Gynecology, 179, 742-749.

D’Alton, M. E., \& Simpson, L. L. (1995). Syndromes in twins. Seminars in Perinatology, 19, 375-386.

Dhall, K., \& Majumdar, S. (1986). Foetal lung maturation in twin gestation. Indian Journal of Medical Research, 83, 422-425. 
Joseph, K. S., Marcoux, S., Ohlsson, A., Liu, S., Allen, A. C., Kramer, M. S., \& Wen, S. W. (2001). Changes in stillbirth and infant mortality associated with increases in preterm birth among twins. Pediatrics, 108, 1055-1061.

Hertz-Picciotto, I., \& Din-Dzietham, R. (1998). Comparisons of infant mortality using a percentile-based method of standardization for birthweight or gestational age. Epidemiology, 9, 61-67.

Kiely, J. (1990). The epidemiology of perinatal mortality in multiple births. Bulletin of the New York Academy of Medicine, 66, 618-637.

Kiely, J. L. (1998). What is the population-based risk of preterm birth among twins and other multiples? Clinical Obstetrics and Gynecology, 41, 3-11.

Kilpatrick, K. J., Jackson, R., \& Croughan-Minihane, M. S. (1996). Perinatal mortality in twins and singletons matched for gestational age at delivery at $>30$ weeks. American Journal of Obstetrics and Gynecology, 174, 66-71.

Kleinbaum, D. G. (1988). Logistic regression: A self learning text. New York: Springer-Verlag New York Incorporated.

Lee, K. I., \& Koval, J. J. (1997). Determination of the best significance level in forward stepwise logistic regression.
Communications in Statistics: Simulation and Computation, 23, 559-575.

Leveno, K. J., Quirk, J. G., Whalley, P. J., Herbert, W. N. P., \& Trubey, R. (1984). Fetal lung maturation in twin gestation. American Journal of Obstetrics and Gynecology, 148, 405-411.

Marviate, M., \& Norman, R. J. (1992). Twins. Clinics in Obstetrics and Gynaecology, 66, 618-637.

Minakami, H., \& Sato, I. (1996). Reestimating date of delivery in multifetal pregnancies. JAMA, 175, 1522-1528.

Mickey, R. M., \& Greenland, S. (1989). The impact of confounder selection criteria on effect estimation. American Journal of Epidemiology, 129, 125-137.

Payne, J. C. (1997). Factors affecting singleton and twin perinatal mortality. Unpublished M.Sc. Thesis, Department of Epidemiology and Biostatistics, University of Western Ontario.

Rooth, G. (1980). Low birthweight revised. Lancet, 1, 639-641.

Williams, K., Hennessy, E., \& Alberman, E. (1996). Cerebral palsy: Effects of twinning, birth weight, and gestational age. Archives of Diseases of Childhood, 75, F178-182. 